\author{
Christos Loupatatzis \\ Sebastian Schindera \\ Jan Gralla \\ Hanno Hoppe \\ Jan Bittner \\ Ralph Schröder \\ Sudesh Srivastav \\ Harald Marcel Bonel
}

\section{Whole-body computed tomography for multiple traumas using a triphasic injection protocol}

Received: 18 September 2007

Revised: 27 October 2007

Accepted: 2 December 2007

Published online: 13 February 2008

(C) European Society of Radiology 2008

C. Loupatatzis $\cdot$ S. Schindera $\cdot$

J. Gralla $\cdot$ H. Hoppe $\cdot$ H. M. Bonel $(\bowtie)$

Institute for Diagnostic,

Interventional and Pediatric Radiology,

Inselspital Berne,

Freiburgstrasse 10,

CH-3010 Berne, Switzerland

e-mail: bonel@gmx.ch

Tel.: +41-31-6322172

Fax: +41-31-6324874

\section{J. Bittner · R. Schröder}

Emergency and Trauma Unit,

University Hospital of Berne - Inselspital,

Freiburgstrasse 10,

CH-3010 Berne, Switzerland

\section{S. Srivastav}

Department of Biostatistics,

Tulane University,

New Orleans, LA, USA

\begin{abstract}
To evaluate a triphasic injection protocol for whole-body multidetector computed tomography (MDCT) in patients with multiple trauma. Fifty consecutive patients (41 men) were examined. Contrast medium (300 $\mathrm{mg} / \mathrm{mL}$ iodine) was injected starting with $70 \mathrm{~mL}$ at $3 \mathrm{~mL} / \mathrm{s}$, followed by $0.1 \mathrm{~mL} / \mathrm{s}$ for $8 \mathrm{~s}$, and by another bolus of $75 \mathrm{~mL}$ at $4 \mathrm{~mL} / \mathrm{s}$. CT data acquisition started $50 \mathrm{~s}$ after the beginning of the first injection. Two experienced, blinded readers independently measured the density in all major arteries, veins, and parenchymatous organs. Image quality was assessed using a five-point ordinal rating scale and compared to standard injection protocols $[n=25$ each for late arterial chest, portovenous abdomen, and MDCT angiography (CTA)]. With the exception of the infrarenal inferior caval vein, all blood vessels were depicted with
\end{abstract}

diagnostic image quality using the multiple-trauma protocol. Arterial luminal density was slightly but significantly smaller compared to CTA $(P<0.01)$. Veins and parenchymatous organs were opacified significantly better compared to all other protocols $(P<0.01)$. Arm artifacts reduced the density of spleen and liver parenchyma significantly $(P<0.01)$. Similarly high image quality is achieved for arteries using the multiple-trauma protocol compared to CTA, and parenchymatous organs are depicted with better image quality compared to specialized protocols. Arm artifacts should be avoided.

Keywords Multiple trauma . Computed tomography $\cdot$ Contrast media $\cdot$ Injection protocol

\section{Introduction}

Whole-body computed tomography (CT), with its technical advances driven mainly by the introduction of multidetector CT systems in the last decade, has become an increasingly valuable tool in the early clinical management of patients with multiple traumas $[1,2]$. The short duration of the examination and its high sensitivity and specificity compared to other clinical or imaging alternatives have contributed to its importance $[3,4]$.

However, to date the CT protocol for multiple-trauma patients is still subject to debate. A dedicated CT protocol must represent the best trade-off between a short acquisi- tion time and maintenance of high diagnostic image quality. Both examination speed and increasing concern about the radiation dose applied [5] necessitate one spiral acquisition instead of multiple different phases.

In addition to the patients' positioning, the administration of contrast media in this group of patients has a critical contribution to diagnostic quality. Different lesions, usually evaluated in different phases of contrast distribution, have to be illustrated: e.g., hematoma and ongoing arterial hemorrhage have to be distinguished, and parenchymal contusion or laceration of organs have to be depicted. A high dose of contrast media is generally accepted for adult multiple-trauma patients [6]. However, the administration 
of contrast medium is difficult because bolus tracking is often inadequate following multiple trauma on account of the circulatory instability present in most cases $[2,6]$.

We investigated a novel triphasic injection protocol with a fixed delay to display all vascular structures and parenchymatous organs simultaneously with high contrast and diagnostic quality. The hypothesis of our paper is that this method of application depicts all relevant anatomy with an image contrast and quality similar to specialized $\mathrm{CT}$ protocols. To evaluate the injection protocol proposed in this paper, both qualitative and quantitative evaluations were performed by two independent readers. In patients with suspected lesions to the arms and shoulders, the arms should not be positioned above the head; we therefore evaluated the influence of artifacts caused by the arms, if positioned lateral to the body, on contrast enhancement of all vascular structures and parenchymatous organs. In addition, the outcome was checked to ensure that lesions had not been missed.

\section{Material and methods}

The present investigation was performed in a level I trauma center, in which computed tomography has become the main imaging investigation tool in the management of patients with multiple trauma. The retrospective study was approved by the institutional review board and was performed according to the regulations of the local ethics committee for retrospective studies.

\section{Equipment and scanning protocol}

The multiple-trauma protocol included CT of the head and cervical spine, which were acquired without intravenous contrast medium and are not evaluated as part of this study. This was followed by one craniocaudal spiral CT acquisition that started at the middle of the seventh cervical vertebrae to exclude supraaortal dissection and ended at the proximal femur. All examinations compared were performed on a 16-slice multidetector row computed tomography system (Sensation 16, Siemens, Forchheim, Germany) with a collimation of $16 \times 1.5 \mathrm{~mm}$ and a reconstruction slice thickness of both 2 and $5 \mathrm{~mm}$. For normal-sized patients, a voltage of $120 \mathrm{kV}$ was used for the "arms-up" protocol. For the "arms-down" protocol and for patients estimated to be more than $120 \mathrm{~kg}$ in body weight, a voltage of $140 \mathrm{kV}$ was applied to compensate for the additional attenuation, especially of the shoulders. The protocol used the CARE dose 4D automatic exposure control (Siemens, Forchheim, Germany) to optimize the current $(\mathrm{mA})$ relative to body attenuation. The reference dose can only be set to one reference value for one acquisition. The reference current was set to $160 \mathrm{~mA}$ for standard chest exams; for all other protocols, it was set to $200 \mathrm{~mA}$. The protocol is performed in all adult patients who present with multiple trauma (two injured body regions of which one is potentially fatal) but with consideration of circumstances of the injury [2].

The contrast medium was administered via an $18 \mathrm{G}$ peripheral access by means of a programmable injection pump using a fixed triphasic injection protocol. For all injections in this study, the same injection device (EnVision, Medrad, Indianola, PA, USA) was used with the same set of afferent tubes. For the injection of contrast media, we used a fixed protocol with a first injection of $70 \mathrm{~mL}$ at a rate of $3 \mathrm{~mL} / \mathrm{s}$ of a contrast medium containing $300 \mathrm{mg} / \mathrm{mL}$ iodine (Ultravist 300, Berlex Laboratories, Montville, NJ, USA), followed by an interval of low-flow-rate contrast injection $(0.1 \mathrm{~mL} / \mathrm{min})$ to prevent collapse of the afferent venous line. Then, another $75 \mathrm{~mL}$ bolus of contrast medium was injected at a rate of $4 \mathrm{~mL} / \mathrm{s}$ (Table 1). Accordingly, the first injection phase lasted $23.3 \mathrm{~s}$, the second phase lasted $8 \mathrm{~s}$, and the third phase lasted $18.7 \mathrm{~s}$. The protocol was based on prior experience with injection speeds in monophasic injection protocols and aims for bolus coherence of the arterial (phase 1) and portal phases (phase 3), while the intermitted 8-s-long second phase supports a timely portal phase. The CT acquisition was started $50 \mathrm{~s}$ after the beginning of the first injection. The body weights and the renal clearances of the patients and their history of possible reactions to contrast material were not known at the time of the examination.

\section{Patients}

Fifty consecutive patients aged $43.5 \pm 21.5$ years (average \pm SD) including 41 men and 9 women were evaluated

Table 1 Injection protocols

\begin{tabular}{|c|c|c|c|c|c|}
\hline & Multiple trauma & $\begin{array}{l}\text { CT } \\
\text { angiography }\end{array}$ & $\begin{array}{l}\text { CTA pulmonary } \\
\text { main stem }\end{array}$ & $\begin{array}{l}\text { Chest CT } \\
\text { late arterial phase }\end{array}$ & $\begin{array}{l}\text { Abdomen } \\
\text { portovenous phase }\end{array}$ \\
\hline Delay (s) & 50 & Trigger & Trigger & 30 & 60 \\
\hline Flow $(\mathrm{mL} / \mathrm{s})$ & Phase $1: 3$, phase $2: 0.1$, phase $3: 4$ & 4 & 4 & 3 & 3 \\
\hline Amount (mL) & Phase 1: 70 , phase $2: 1$, phase $3: 75$, total: 146 & 120 & 100 & 80 & 120 \\
\hline
\end{tabular}

For all injections, a contrast medium containing $300 \mathrm{mg} / \mathrm{mL}$ iodine was used. The injection device could not inject an additional saline flush to empty the afferent tubes; therefore, $4 \mathrm{~mL}$ of contrast media remained in the tube (total $150 \mathrm{~mL}$ ) 
retrospectively (November 2003 until January 2004). To evaluate the effect of the position of the arms, we used a subgroup of 25 consecutive patients with the arms elevated above the head and compared these patients to another consecutive 25 patients with the arms positioned at the side of the body trunk. If there is clinical suspicion of a lesion to the shoulder girdle or cervical spine, the arms are positioned alongside the body. Putting the hands above the head is an additional step in patient handling because the preceding $\mathrm{CT}$ of the skull and cervical spine requires the hands and arms to be positioned caudally [4].

In addition, similar consecutive patient cohorts were sampled from the same CT system with standard protocols for pulmonary embolism, a standard late-arterial-phase chest protocol (mainly tumor staging protocols), abdominal exams in the portovenous phase (mainly tumor staging and exclusion of abscess) and whole-body computed angiography (CTA) (mainly for aneurysms, bleeding and planning of interventions). Table 1 gives the details of the injection protocols. There was no significant age difference between the two subgroups tested for the preferred position of the arms $(P=0.86)$. Patient groups examined with standard protocols were on average 16 years older $(59.7 \pm 15.2)$ than those who underwent the multiple-trauma protocols.

\section{Evaluation}

All evaluation criteria were evaluated on a standard PACS system (Easyvision, Philips, The Netherlands) by two radiologists with 4 years of experience in computed tomography (CL and SS) in independent sessions. Image quality served as the primary end point and was evaluated on an ordinal scale ranging from 5 (maximum image quality), 4 (good image quality), 3 (still diagnostic image quality), 2 (image quality inadequate for diagnostic evaluation), to 1 (image quality does not allow accurate diagnosis) $[8,9]$. Densities were measured in all patients and served as secondary end-points and were determined by measuring vessel attenuation (in Hounsfield units, $\mathrm{HU}$ ) in regions of interest (ROIs) that were placed in the ascending, descending, and abdominal aorta and covered a maximum of the vessel lumen $\left(<1 \mathrm{~cm}^{2}\right)$. Ten regions including both arteries and veins were measured in each patient, and in addition, standard deviation of background noise was assessed $[6,7]$.

The densities of parenchymal organs were measured by placing ROIs in the right and left lobes of the liver, in the spleen, and in the kidneys covering the largest possible area within the parenchyma but without including vessels, artifacts, or lesions (in the slice measured and in both adjacent slices, $<3 \mathrm{~cm}^{2}$ ) [6]. Density was measured in the lesion of patients with liver and spleen lacerations $(n=10)$ and compared to artifacts caused by the arms at the side of the body $(n=25)$.

All patients were followed up by physicians of the Departments of Radiology, Emergency, and Intensive Care.
Follow-up examinations, discharge reports, and outcome were checked for any additional findings (JB and RS).

\section{Biostatistics}

All statistical analyses were performed using SAS statistical software, version 9.1 (SAS Institute, Cary, NC, USA), and all results were tested at a 5\% level of significance. Data were tested for normal distribution, and the Student $t$-test (or Wilcoxon signed rank test) was used as a parametric (or nonparametric) technique for comparing two groups. The analysis of variance (ANOVA) method was used to compare more than two groups for studied variables. Interobserver variability was calculated and analyzed using the Cohen $\mathrm{K}$ test. Interobserver agreement was scored as poor ( $K=0.1$ to 0.4$)$, fair $(K=0.41$ to 0.6 ), moderate ( $K=0.61$ to 0.8$)$ or excellent ( $K=0.81$ to 1.0$)$ [10].

\section{Results}

Background noise was measured for all patients of the multiple-trauma protocols and standard protocols and was not significantly different (average -996.1 Hounsfield units, standard deviation $8.4 ; P>0.12$ ).

\section{Vessels}

Table 2 summarizes all vessels measured. Vascular injury was diagnosed in $n=11$ patients. Image quality was rated to high diagnostic levels for all vessels except the inferior cava and portal vein. Artifacts caused by having the arms positioned lateral to the trunk deteriorated the image quality significantly in the pulmonary artery and all veins $(P<0.01)$.

The ascending and descending aorta showed similar densities with a clear tendency in all protocols (Fig. 1): the multiple-trauma protocol without arm artifacts and the CTA depicted the thoracic aorta with the highest density. In spite of the small but significant difference between the CTA protocols and the protocol for multiple trauma $(P<$ $0.01)$, image quality was rated to a similarly high level $(P=$ 0.31 , Table 2 ). The intra-arterial density obtained using the multiple-trauma protocol was significantly better than all other protocols except CTA $(P<0.01)$.

The pulmonary artery was opacified best by CTA and the dedicated protocol without any significant difference $(P=0.64)$. Both multiple-trauma protocols depicted the pulmonary artery well, and significantly better than a standard chest $\mathrm{CT}$ protocol $(P=0.0001)$. However, artifacts from the arms already caused a significant deterioration of luminal density $(P<0.001)$.

The abdominal aorta and iliac arteries were rated very similarly in density levels and image quality. Despite a 
Table 2 Measurements (median \pm SD) of vessels evaluated in this study

\begin{tabular}{|c|c|c|c|c|c|c|c|}
\hline & & $\begin{array}{l}\text { Multiple } \\
\text { trauma, } \\
\text { arms up }\end{array}$ & $\begin{array}{l}\text { Multiple } \\
\text { trauma, } \\
\text { arms down }\end{array}$ & CTA & $\begin{array}{l}\text { CTA } \\
\text { pulmonary } \\
\text { main stem }\end{array}$ & Chest CT & $\begin{array}{l}\text { Abdomen } \\
\text { portovenous } \\
\text { phase }\end{array}$ \\
\hline \multirow[t]{2}{*}{ Ascending aorta } & Density (HU) & $272.8 \pm 70.7$ & $255.2 \pm 48.0$ & $314.6 \pm 78.9$ & $279.2 \pm 90.3$ & $203.1 \pm 47$ & - \\
\hline & VAS & $4.7 \pm 0.5$ & $4.7 \pm 0.5$ & $4.7 \pm 0.5$ & $4.6 \pm 0.5$ & $4.6 \pm 0.7$ & - \\
\hline \multirow[t]{2}{*}{ Descending aorta } & Density (HU) & $279.1 \pm 68.5$ & $254.5 \pm 53.7$ & $299.8 \pm 74.7$ & $259.3 \pm 89.9$ & $199.3 \pm 43.3$ & - \\
\hline & VAS & $4.7 \pm 0.5$ & $4.7 \pm 0.5$ & $4.7 \pm 0.5$ & $4.6 \pm 0.5$ & $4.6 \pm 0.7$ & - \\
\hline \multirow[t]{2}{*}{ Pulmonary artery main stem } & Density (HU) & $216.2 \pm 58.8$ & $201.8 \pm 52.3$ & $307.0 \pm 89.1$ & $311.2 \pm 122.7$ & $158.7 \pm 64.1$ & - \\
\hline & VAS & $4.2 \pm 0.8$ & $3.7 \pm 0.7$ & $5.0 \pm 0.2$ & $4.9 \pm 0.4$ & $3.0 \pm 0.9$ & - \\
\hline \multirow[t]{2}{*}{ Abdominal aorta suprarenal } & Density (HU) & $232.0 \pm 68.9$ & $218.3 \pm 53.3$ & $307.7 \pm 75.7$ & - & - & $131.7 \pm 23.0$ \\
\hline & VAS & $4.6 \pm 0.5$ & $4.5 \pm 0.6$ & $4.7 \pm 0.3$ & - & - & $3.2 \pm 0.6$ \\
\hline \multirow[t]{2}{*}{ Abdominal aorta infrarenal } & Density (HU) & $229.9 \pm 68.0$ & $221.9 \pm 52.7$ & $315.6 \pm 77.2$ & - & - & $132.7 \pm 22.5$ \\
\hline & VAS & $4.6 \pm 0.6$ & $4.5 \pm 0.6$ & $4.6 \pm 0.3$ & - & - & $3.2 \pm 0.6$ \\
\hline \multirow[t]{2}{*}{ Common iliac artery ${ }^{a}$} & Density (HU) & $226.4 \pm 69.3$ & $212.4 \pm 50.7$ & $310.2 \pm 78.1$ & - & - & $136.2 \pm 21.8$ \\
\hline & VAS & $4.6 \pm 0.6$ & $4.4 \pm 0.7$ & $4.6 \pm 0.3$ & - & - & $3.1 \pm 0.6$ \\
\hline \multirow[t]{2}{*}{ IVC between kidney and bifurcation } & Density (HU) & $92.7 \pm 34.8$ & $91.8 \pm 29.3$ & $49.4 \pm 22.4$ & - & - & $82.4 \pm 21.2$ \\
\hline & VAS & $3.4 \pm 1.0$ & $3.0 \pm 0.8$ & $1.9 \pm 0.4$ & - & - & $3.3 \pm 0.7$ \\
\hline \multirow[t]{2}{*}{ IVC between liver and kidneys } & Density (HU) & $139.0 \pm 36.8$ & $131.7 \pm 36.1$ & $79.0 \pm 34.4$ & - & - & $110.2 \pm 21.6$ \\
\hline & VAS & $2.8 \pm 1.1$ & $2.2 \pm 1.2$ & $1.5 \pm 0.2$ & - & - & $2.7 \pm 0.7$ \\
\hline \multirow[t]{2}{*}{ Portal vein } & Density (HU) & $184.5 \pm 33.4$ & $164.8 \pm 41.3$ & $78.9 \pm 24.4$ & - & - & $124.7 \pm 21.5$ \\
\hline & VAS & $4.4 \pm 0.6$ & $3.5 \pm 0.9$ & $2.3 \pm 0.7$ & - & - & $4.3 \pm 0.5$ \\
\hline
\end{tabular}

$H U$ Hounsfield units, VAS visual assessment scale, $I V C$ inferior vena cava, $C T A$ computed tomography performed as an angiography protocol ${ }^{a}$ Measurements from the left and right common iliac arteries were not significantly different; therefore, averages of both sides are given

significantly smaller density compared to CTA $(P<0.01)$, both multiple-trauma protocols were rated to the same diagnostic level and were significantly better compared to an abdominal CT obtained in the portovenous phase $(P<0.01)$.

The inferior cava cranial and caudal to the kidneys and the portal vein showed significantly better density compared to CTA and the standard abdominal protocol $(P<0.001)$. Artifacts from the arms diminished the opacification of the portal vein significantly $(P<0.001)$, but did not play a significant role for the inferior cava $(P=0.24)$. The inferior cava between the liver and kidneys was rated with a significantly better diagnostic level $(P<0.01)$ than the more caudal inferior caval vein, which was not rated to a diagnostic level in any of the protocols. An additional CT acquisition with a longer delay was not deemed necessary for any of the 50 patients.

\section{Parenchymatous organs}

Density measurements in all organs using both multipletrauma protocols were significantly better compared to CTA and standard abdomen protocols $(P<0.001)$ (Figs. 2 and 3; Table 3). Arm artifacts degraded image quality to a level that was rated significantly worse compared to the arms-up protocol and the standard abdomen protocol $(P<$
0.001) for the liver and spleen; however, image quality did not change significantly for the kidneys.

Artifacts caused by the arms showed a significantly lower density $(88.6 \pm 27.3)$ compared to normal spleen or liver $(P<0.01)$. Hematoma within the organ was displayed with a significantly lesser density $(52.8 \pm 15.5)(P<0.01)$ compared to artifacts. Hematoma was not confused with these artifacts in any of the patients. However, the image quality of the liver and spleen was scored significantly lower in patients with arm artifacts compared to the patient group without arm artifacts; image quality of the kidneys was not influenced by the position of the arms.

\section{Interreader correlation}

Interreader correlation was excellent or moderate for the following [10]: abdominal aorta $(\mathrm{K}=0.66)$, portal vein $(K=0.66)$, spleen $(K=0.68)$, pulmonary trunk $(K=$ $0.92)$ and artifacts $(\kappa=0.61)$. The kidneys $(\kappa=0.53)$, thoracic aorta $(K=0.52)$, the liver $(K=0.43)$, pelvic arteries $(K=0.44)$ and the inferior cava cranial to the kidneys $(K=0.48)$ were reported with a fair interreader correlation. The infrarenal cava $(\kappa=0.18)$ was evaluated with only a poor correlation between the two readers, which also reflects that the image quality did not reach a diagnostic level for this structure. 
Fig. 1 A 46-year-old male patient with multiple trauma after a motorbike accident. The traumatic aortic rupture in the descending aorta is conspicuous on both axial images and sagittal reconstructions (black arrows). In addition, hepatic rupture can be well appreciated in axial images from the same acquisition (open arrow in lower left image). A stent graft was positioned in the descending aorta $13 \mathrm{~h}$ later, covering the lesion, and the abdominal hematoma (*) progressed during the first $24 \mathrm{~h}$ after trauma but resolved later without complication

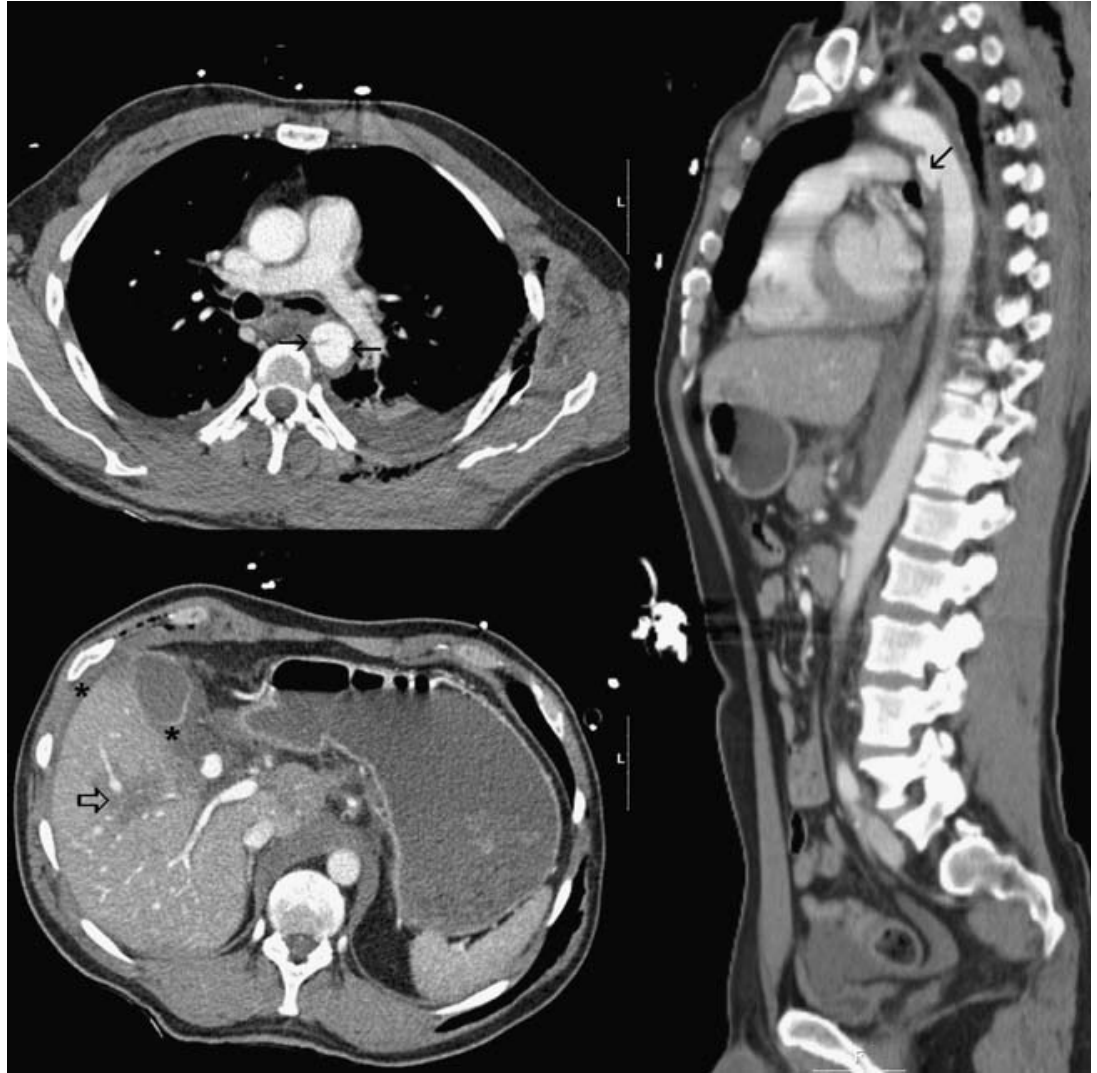

\section{Outcome}

All patients were followed-up until discharge by physicians of the emergency and intensive care unit for 22-288 days. Additional imaging studies were available in all patients (computed tomography: $n=23$; ultrasound: $n=38$, plain $\mathrm{x}-$ rays: $n=50$ ). In addition, patient charts were evaluated retrospectively. There were no missed diagnoses reported that would have influenced patient management in any way.

\section{Discussion}

Various studies have been published with regard to optimizing liver and splanchnic vessel opacification [7, 11-14]; these

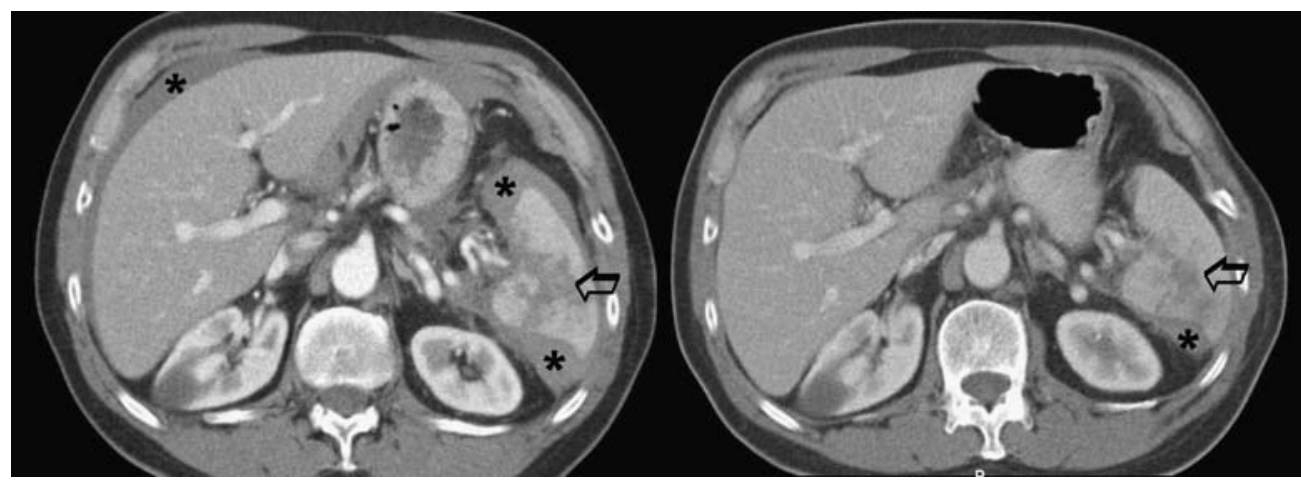

Fig. 2 Traumatic laceration of the spleen in a 63-year-old male patient. The triphasic injection protocol (on the left) shows the laceration of the spleen with hilar involvement very well (arrows). Opacification of the liver vessels, the aorta, and the splenic artery is very conspicuous. The hematomas of the spleen and liver are very well delineated $(*)$. The standard protocol of the abdomen in the portovenous phase (5.5 days later, on the right) shows the decrease in the perisplenic $(*)$ and perihepatic hematoma. Image contrast using the same window setting is significantly worse; vessels and parenchymatous organs, as well as the hematoma, are depicted with less visibility. Anatomy appears less delineated 


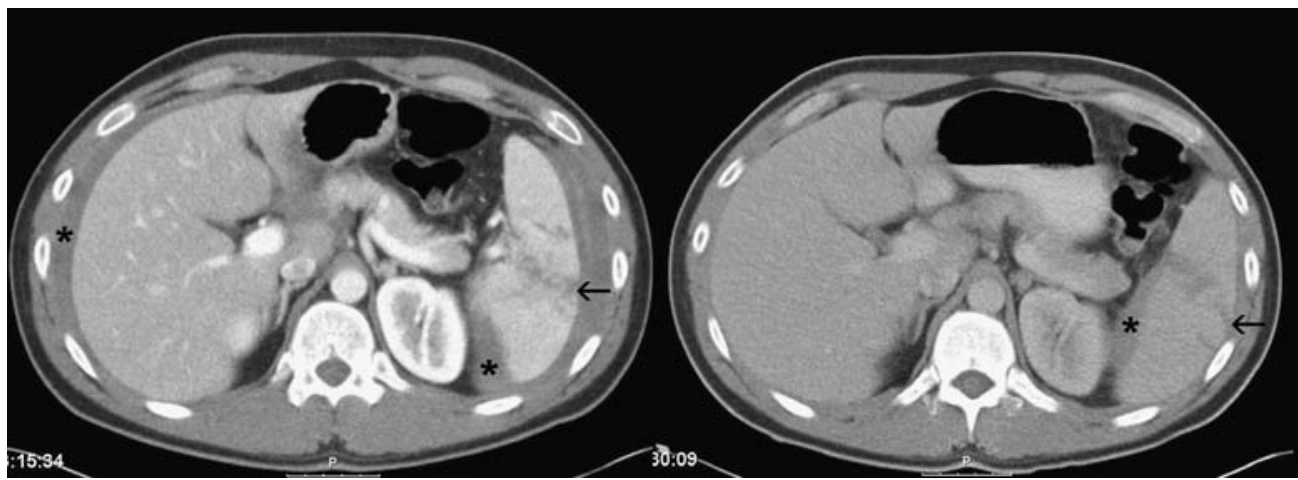

Fig. 3 Laceration of the spleen in a 33-year-old male patient. The triphasic protocol shows exquisite anatomy of all the intrahepatic vessels, the splenic vein, and hilar arterial branches (image on the left). The kidney, liver, and spleen are also shown with a high contrast compared to the hematoma $(*)$. On the right, the standard protocol (6 days later) with its fixed delay is too slow. However, the

show that injection protocols with more than just one injection phase are advantageous compared to protocols that use a single injection phase. Bolus tracking is recommended for the upper abdomen [15], but not in patients with multiple trauma because of the circulatory instability present in most cases [2,6]. Instead, a fixed time delay using an amount of $150 \mathrm{~mL}$ seems to be the method of choice $[2,6]$. This already large amount of contrast media could limit its further use for angiographic interventions; however in the future, the use of a 64-detector scanner could decrease the amount of contrast media needed.

The triphasic protocol that we have used for this study tries to incorporate all aspects that have been reported to be of advantage: a multiphase injection protocol using optimized amounts of contrast media at optimized injection speeds, a fixed time delay, and only a slightly larger amount of i.v. contrast media compared to standard routine protocols. The results of our study show that the contrast enhancement using the triphasic protocol reaches sufficient laceration of the spleen (arrows) can still be appreciated; the decrease in perihepatic and perisplenic hematoma is confirmed, but depicted with a significantly lower contrast compared to the multiple-trauma protocol on the left. Also, organ opacification in the standard protocol on the right is notably worse compared to Fig. 2; however, in both patients a fixed delay of $60 \mathrm{~s}$ was used

diagnostic quality for the dedicated requirements in the evaluation of multiple-trauma patients, and even exceeded the quality of standard protocols in some aspects.

\section{Vessel enhancement}

Opacification of the major blood vessels of the chest, abdomen, and pelvis achieved high diagnostic levels. There were no diagnoses missed in any patients with vascular injury. Opacification of both the thoracic and abdominal aorta was slightly, but significantly, lower in the triphasic protocol compared to CTA, which can be considered a standard of reference [11]. However, this reduced opacification of the arterial lumen did not affect the qualitative evaluation. With the exception of the inferior cava and its branches caudal to the kidneys, which would require a much longer delay, all veins and arteries of importance were depicted with better than

Table 3 Measurements (median \pm SD) of abdominal parenchymatous organs

\begin{tabular}{llllll}
\hline Organ & & $\begin{array}{l}\text { Multiple trauma, } \\
\text { arms up }\end{array}$ & $\begin{array}{l}\text { Multiple trauma, } \\
\text { arms down }\end{array}$ & CT angiography & $\begin{array}{l}\text { Abdomen } \\
\text { portovenous phase }\end{array}$ \\
\hline Liver $^{\mathrm{a}}$ & Density (HU) & $101.7 \pm 16.9$ & $108.7 \pm 17.3$ & $65.4 \pm 14.0$ & $94.1 \pm 16.4$ \\
& VAS & $4.2 \pm 0.6$ & $3.2 \pm 0.6$ & $2.0 \pm 0.1$ & $4.1 \pm 0.5$ \\
Spleen & Density (HU) & $144.0 \pm 19.6$ & $137.9 \pm 28.2$ & $100.5 \pm 16.6$ & $104.1 \pm 14.3$ \\
& VAS & $4.5 \pm 0.5$ & $3.5 \pm 0.9$ & $2.3 \pm 0.7$ & $4.3 \pm 0.5$ \\
Right kidney & Density (HU) & $200.6 \pm 40.2$ & $201.0 \pm 39.7$ & $153.8 \pm 38$ & $154.2 \pm 28.6$ \\
& VAS & $4.0 \pm 0.3$ & $4.1 \pm 0.4$ & $3.2 \pm 0.6$ & $3.6 \pm 0.5$ \\
Left kidney $^{b}$ & Density (HU) & $206.0 \pm 39.7$ & $211.0 \pm 34.3$ & $147.0 \pm 46.6$ & $151.8 \pm 28.8$ \\
& VAS & $4.0 \pm 0.3$ & $4.1 \pm 0.4$ & $3.1 \pm 0.7$ & $3.5 \pm 0.6$ \\
\hline
\end{tabular}

$H U$ Hounsfield units, $V A S$ visual assessment scale

${ }^{a}$ Liver assessments were originally done for the left and right lobes separately. However, there were not any significant differences between the two liver lobes, and therefore only the average measurements and ratings are given in this table

${ }^{\mathrm{b}}$ Measurements given for the kidneys were performed in the cortex 


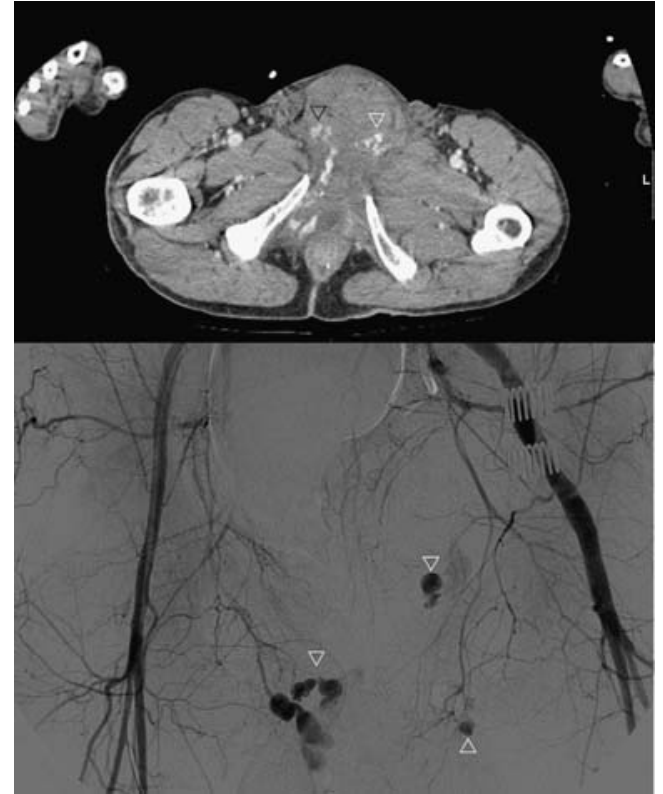

Fig. 4 A 56-year-old male patient with pelvic trauma following a car accident. The axial CT shows a simultaneous good arterial and venous opacification of the femoral arteries and veins. The extravasation can be appreciated very well, and subtraction angiography verifies the bleeding sites of branches of the pudendal arteries (arrowheads)

diagnostic image quality. No additional imaging in a later venous phase was deemed necessary in any of our patients; this is another indicator of sufficient diagnostic quality. Thus this protocol offers the potential to reduce cumulative radiation dosage by avoiding additional sequences. In our experience, the early injection phase of our protocol also helps identify extravasation (Fig. 4). Indeed the opacification during the first phase is so efficient that even bleeding from spongy bone can be appreciated (Fig. 5).
If the arm cannot be positioned above the head, e.g., in cases of shoulder injury [15-17], artifacts are inevitable. In particular, the bones of the shoulders, arms, and hands cause reduction in the luminal opacification with a spiral aspect. These absorption artifacts from the arms and hands decrease image quality significantly, especially in the pulmonary artery and portal vein. While diagnostic image quality is still obtained in the pulmonary artery, it is decreased in the portal vein to a nondiagnostic level in a substantial proportion of the patients. The recommendation, therefore, must be to avoid these artifacts whenever possible.

The combined arterial and venous enhancement was sufficient to detect vascular pelvic bleeding (Fig. 5). However, more complex injection methods could be beneficial to make arterial and venous opacification in the lower abdomen and pelvis more uniform [11]. Interindividual differences in the backflow of contrast medium in the legs, however, are dependent on many more factors than just heart rate and circulatory time, e.g., in cases of peripheral arterial occlusive disease, so this aspect is especially challenging.

\section{Parenchymal enhancement}

The image quality of all parenchymatous organs significantly benefits from a triphasic injection protocol compared to all other regimens; this confirms the results of other groups [19]. In particular, the spleen and kidneysboth at risk in a multiple-trauma patient-are opacified very well compared to standard protocols (Figs. 1, 2, and 3) [20]. Our protocol demonstrates both the kidney cortex and the medulla (Figs. 2 and 3) and results in more homogeneous enhancement of the whole organ. Lacerations and vessel injuries can be detected at the same time. If only a single early phase of enhancement is used, as in the

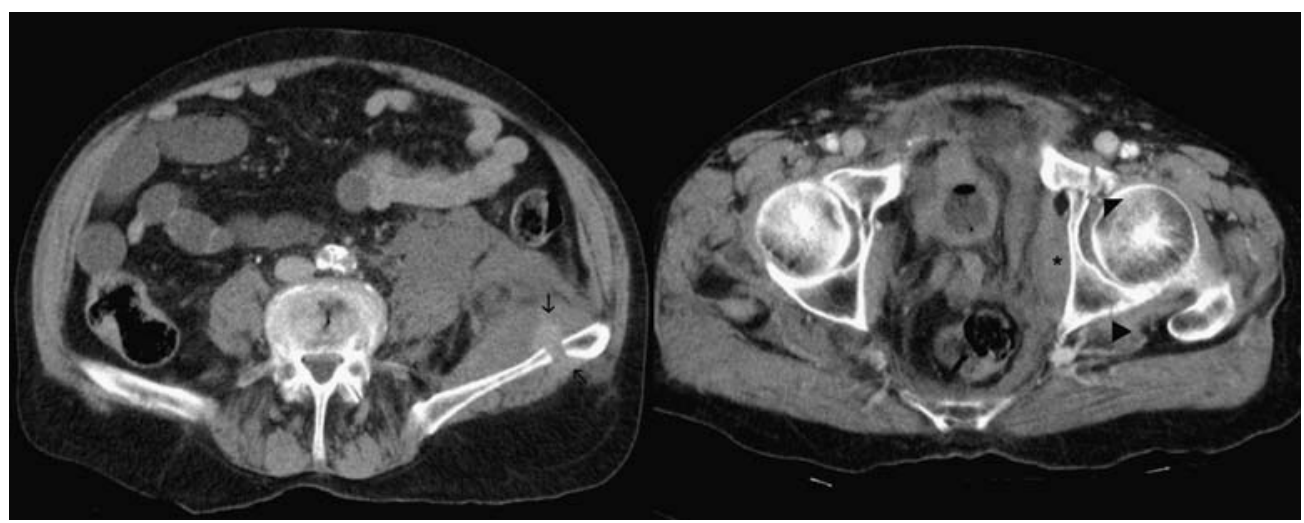

Fig. 5 Pelvic fracture in an 85-year-old man. On the left side of the patient, the extravasation of contrast medium from the spongy bone is visible due to the delayed phase from the first injection (black arrows, left image). Note that the hematoma in the psoas muscle is less dense in comparison. Caudally the acetabular fracture (black arrowheads in the image on the right) and the hematoma (*) without extravasation of contrast medium can be seen. This helps to identify the source of bleeding in unstable patients. Note the simultaneous good arterial and venous opacification of the common femoral vessels; peripheral arterial occlusive disease leads to the reduced opacification of the right common femoral vein, caused by a delayed backflow 
compared CTA protocol, only vessels and the cortex are shown well; a later portovenous phase presents cortex versus calices with reduced parenchymal opacification. The readers in our study reported a simpler evaluation of the kidneys due to the improved homogeneous enhancement of the medulla and higher contrast of the cortex at the same time, giving them a better anatomic presentation of the kidneys (Fig. 3). However, an unenhanced study, which might be helpful to detect intraparenchymal hematoma that may be isodense with normal renal parenchyma [21], was not performed. Delayed imaging to detect pelvicalyceal or ureteric disruption was not performed in any patient; however, follow-up ultrasound exams did not reveal such an injury in any patient.

Despite a significant deterioration in image quality and organ density, artifacts from the arms were not a diagnostic problem in any of the patients. One reason might be that the density of artifacts was significantly higher than hematoma. In addition, the morphology of the artifacts was so obviously different from hematoma that it posed a smaller problem to the reporting radiologist than originally thought. Follow-up CT of our patients using dedicated protocols have not shown a missed hematoma or organ laceration; however, very small lesions might have been missed but are probably not of any clinical relevance. On the other hand, the time savings of not repositioning the arms after the examination of the head and cervical spine is so small that there must be a clinical reason, such as a traumatic injury to the upper chest or shoulder girdle, that the arms are not brought towards the head $[16,17]$. In our experience, the image quality of the kidneys was not affected as much as that of the liver and spleen, so it is mainly these organs that require special attention.

\section{Study limitations}

Some limitations of our study must be addressed. The majority of our results reached a statistically significant level. However, due to the limited number of patients included in this study, these figures are to a certain extent prone to small number effects. Given the large variation in the effects of trauma, a clinical follow-up in 50 patients is also of limited value. However, since the evaluation 3 years ago, we have used the multiple-trauma protocol without any change and have not seen or had a report of a missed diagnosis due to insufficient vessel or organ enhancement on our regular interdisciplinary boards, which serve as an important quality control for radiologists, anesthesiologists, and physicians from the emergency and intensive care units.

Whether more complex injection algorithms using test boli for optimization [18] would be beneficial for a multiple-trauma protocol can only be speculated; also, the diagnostic value of a higher iodine concentration has not yet been examined in multiple-trauma patients [19, 22]. Finally, our injection device is not able to perform an additional saline flush, e.g., to create better bolus coherence that would be of interest in the third injection phase. We suppose that better bolus coherence could improve image quality.

On the basis of these findings, we suggest that our protocol obviates the need for additional acquisitions of portovenous phases in the vast majority of patients with multiple traumas, with the exception of lesions to the urinary tract: our impression is that late phases in which the calices are opacified might be more sensitive than follow-up with ultrasound, however we cannot derive supporting data from our present study. Still, our injection protocol potentially reduces cumulative radiation dosage in multiple-trauma patients, who are often young. A single CT data acquisition following intravenous contrast medium also means less time is needed for additional acquisitions, for the reporting of these additional images, and for communication of potential new diagnoses from late-phase CT studies to the referring physician. This saves important time in the first and "golden" hour of patient management [23].

\section{Conclusion}

The injection protocol proposed for MDCT examination of patients with multiple trauma results in a significantly improved presentation of parenchymatous organs of the upper abdomen with a simultaneous presentation of the major arteries to the same diagnostic level as a CTA protocol. The follow-up of patients revealed that no major diagnoses were missed during emergency evaluation. Image quality deteriorated to a major extent if the arms had to be positioned alongside the body; therefore, the arms should be positioned at the level of the head and neck whenever possible. Using a triphasic injection protocol obviates the need for additional arterial or portal phase acquisitions in the vast majority of patients by combining these two phases, and thus potentially reduces cumulative radiation dosages and examination costs.

\section{References}

1. Linsenmaier U (1998) Disputed concept in polytrauma: whole-body spiral CT as primary diagnosis. Röfo 168:306
2. Linsenmaier U, Krotz M, Hauser H, Rock C, Rieger J, Bohndorf K, Pfeifer KJ, Reiser M (2002) Whole-body computed tomography in polytrauma: techniques and management. Eur Radiol 12:1728-1740
3. Gralla J, Spycher F, Pignolet C, Ozdoba C, Vock P, Hoppe H (2005) Evaluation of a 16-MDCT scanner in an emergency department: initial clinical experience and workflow analysis. AJR 185:232-238 
4. Hoppe H, Vock P, Bonel HM, Ozdoba C, Gralla J (2006) A novel multipletrauma CT-scanning protocol using patient repositioning. Emerg Radiol 13:123-128

5. Wedegartner U, Lorenzen $M$, Nagel HD, Weber C, Adam G (2004) Diagnostic imaging in polytrauma: comparison of radiation exposure from whole-body MSCT and conventional radiography with organ-specific CT. Röfo 176:1039-1044

6. Rademacher G, Stengel D, Siegmann S, Petersein J, Mutze S (2002) Optimization of contrast agent volume for helical $\mathrm{CT}$ in the diagnostic assessment of patients with severe and multiple injuries. J Comput Assist Tomogr 26:113-138

7. Awai K, Imuta M, Utsunomiya D, Nakaura T, Shamima S, Kawanaka K, Hori S, Yamashita Y (2004) Contrast enhancement for whole-body screening using multidetector row helical CT: comparison between uniphasic and biphasic injection protocols. Radiat Med 22:303-309

8. Jäger L, Bonel H, Liebl M, Srivastav S, Arbusow V, Hempel M, Reiser M (2005) $\mathrm{CT}$ of the normal temporal bone: comparison of multi- and single-detector row CT. Radiology 235:133-141

9. Bonel HM, Jager L, Frei KA, Galiano S, Srivastav SK, Flohr T, Reiser MF, Dinkel HP (2005) Optimization of MDCT of the wrist to achieve diagnostic image quality with minimum radiation exposure. AJR 185:647-654
10. Landis JR, Koch GG (1977) An application of hierarchical kappa-type statistics in the assessment of majority agreement among multiple observers. Biometrics 33:363-374

11. Bae KT, Tran HQ, Heiken JP (2000) Multiphasic injection method for uniform prolonged vascular enhancement at CT angiography: pharmacokinetic analysis and experimental porcine model. Radiology 216:872-880

12. Zandrino F, Curone P, Benzi L, Musante F (2003) Value of an early arteriographic acquisition for evaluating the splanchnic vessels as an adjunct to biphasic CT using a multislice scanner. Eur Radiol 13:1072-1079

13. Kirkpatrick ID, Kroeker MA, Greenberg HM (2003) Biphasic CT with mesenteric CT angiography in the evaluation of acute mesenteric ischemia: initial experience. Radiology 229:91-98

14. Chung YE, Kim KW, Kim JH, Lim JS, Oh YT, Chung JJ, Kim MJ (2006) Optimal delay time for the hepatic parenchymal enhancement at the multidetector CT examination. J Comput Assist Tomogr 30:182-188

15. Mehnert F, Pereira PL, Trubenbach J, Kopp AF, Claussen CD (2001) Automatic bolus tracking in monophasic spiral CT of the liver: liver-to-lesion conspicuity. Eur Radiol 11:580-584

16. Benneker LM, Bonel H, Zumstein MA, Exadakytlos A (2007) A novel multiple-trauma CT-scanning protocol using patient repositioning may increase risks of iatrogenic injuries. Emerg Radiol $13: 349-351$
17. Hoppe H, Gralla J (2007) Invited comment on the work of Benneker et al. "A novel multiple-trauma CT-scanning protocol using patient repositioning may increase risks of iatrogenic injuries". Emerg Radiol 13:353

18. Bae KT, Tao C, Gurel S, Hong C, Zhu F, Gebke TA, Milite M, Hidebolt CF (2007) Effect of patient weight and scanning duration on contrast enhancement during pulmonary multidetector CT angiography. Radiology 242:582-589

19. Suzuki H, Oshima H, Shiraki N, Ikeya C, Shibamoto Y (2004) Comparison of two contrast materials with different iodine concentrations in enhancing the density of the aorta, portal vein, and liver at multi-detector row CT: a randomized study. Eur Radiol 14:2099-2104

20. Park SJ, Kim JK, Kim KW, Cho KS (2006) MDCT findings in renal trauma. AJR 187(5):1146

21. Tunaci A, Yekeler E (2004) Multidetector row CT of the kidneys. Eur J Radiol 52:53-66

22. Engeroff B, Kopka L, Harz C, Grabbe E (2001) Impact of different iodine concentrations on abdominal enhancement in biphasic multislice helical CT (MS-CT). Röfo 173:938-941

23. Blow O, Magliore L, Claridge JA, Butler K, Young YS (1999) The golden hour and silver day: detection and correction of hypoperfusion within 24 hours improves outcome from major trauma. J Trauma 27:964-969 